\title{
23 Erst kommt das Fressen, dann die Moral - Geordnete Arbeitsverhältnisse als Voraussetzung, sich mit ethischen Fragen angemessen zu befassen
}

\author{
Michael Gommel
}

\begin{abstract}
Eine wichtige Grundlage der guten Patientenversorgung ist die angemessene Beschäftigung mit der ethischen Dimension des medizinischen Handelns durch die am Behandlungsprozess Beteiligten. Eine der Bedingungen für das Gelingen dieser ethischen Reflexion im klinischen Alltag kann mit einem bereits in den 1950er-Jahre erdachten und später weiterentwickelten Modell zur Integration von Individuum und Organisation beschrieben werden. Ethische Reflexion kann als menschliches Bedürfnis verstanden werden, das in der Lage ist, intrinsische Motivationskraft zu entfalten. Im Folgenden werden einige Annahmen, die auf die Maslowsche Theorie der menschlichen Bedürfnisse zurückgehen, in ihrer Bedeutung für das Gelingen ethischer Reflexion im klinischen Alltag erläutert.
\end{abstract}

\subsection{Bedürfnisse motivieren zum Handeln}

Mit dem Aufleben der Human-Ressourcen-Ansätze in der Mitte des 20. Jahrhunderts wurde erkannt, dass Menschen auch in Arbeitszusammenhängen von ihren Bedürfnissen beeinflusst werden, und dass diese Bedürfnisse Menschen zum Handeln motivieren. Solche Bedürfnisse - Vorstellungen, Wünsche, Hoffnungen und Erwartungen im Zusammenhang mit der Arbeit - gibt es in allen Arten von Arbeitssituationen, so auch in Kliniken. Die Bedürfnisse der Menschen werden dabei nicht als gleichberechtigt wahrgenommen, sondern können hinsichtlich der Dringlichkeit ihrer Befriedigung in Gestalt einer Bedürfnispyramide [Maslow 1954] gruppiert werden (s. Abb. 14). Je höher ein Bedürfnis in der Pyramide steht, desto später tritt es in der Entwicklung des Individuums auf, und umso größer ist seine individuelle und soziale Gestaltungsbreite. 


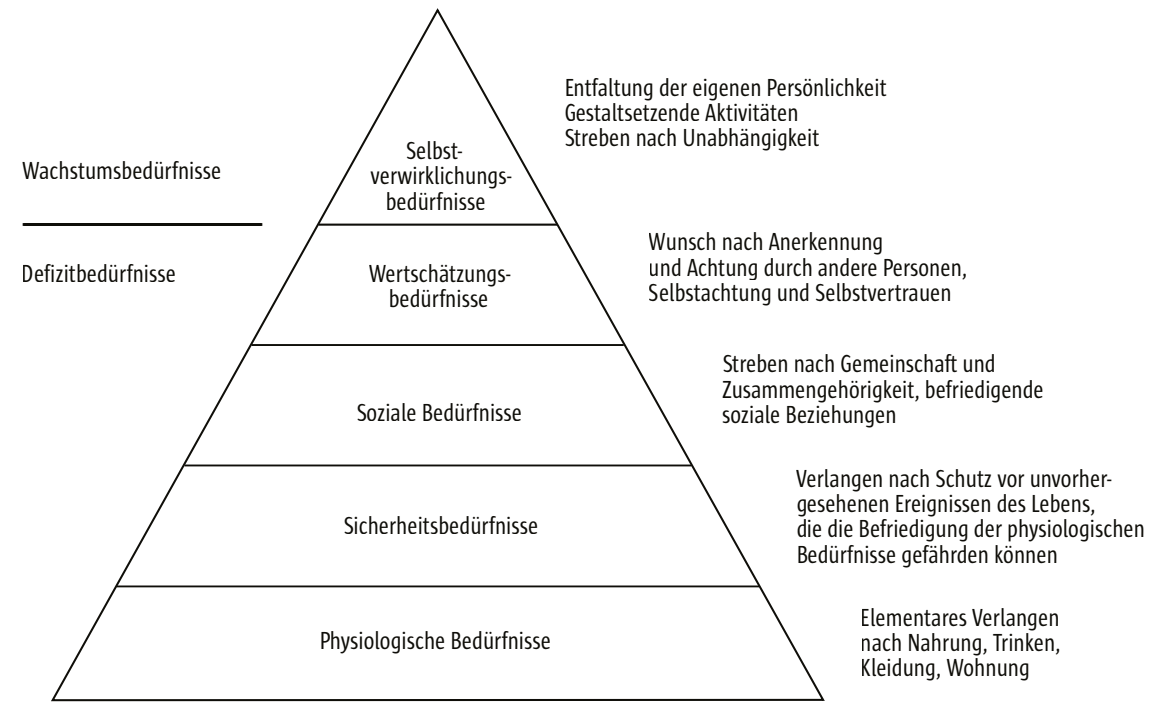

Abb. 14 Maslowsche Bedürfnispyramide

Zwei Prinzipien helfen, die Motivation zur Stillung der Bedürfnisse zu verstehen:

- Das Defizitprinzip besagt, dass nur unbefriedigte Bedürfnisse uns zu entsprechendem Handeln anregen.

- Das Progressionsprinzip drückt aus, dass menschliches Handeln durch das hierarchisch niedrigste, unbefriedigte Bedürfnis motiviert wird.

Sobald dieses Bedürfnis durch entsprechendes Handeln befriedigt ist, geht von ihm kein Handlungsanreiz mehr aus. Es wird das nächst höhere unbefriedigte Bedürfnis aktiviert, das wiederum seine intrinsische Motivationskraft entfaltet. Bei den physiologischen Bedürfnissen wird dabei nicht die aktuelle Befriedigung eines drängenden Bedürfnisses (z. B. Hunger) verstanden, sondern die dauernde Sicherstellung der Möglichkeit (z. B. sich mit Nahrungsmitteln versorgen zu können). Die Bedürfnisse, deren Unbefriedigtheit uns Unbehagen bereitet, nennt Maslow Defizitbedürfnisse; sie können vollständig gesättigt werden. Ihre Befriedigung errichtet, sichert und festigt das Selbst im Zustand der Abhängigkeit von äußeren Faktoren.

Die Befriedigung eines Selbstverwirklichungsbedürfnisses hingegen öffnet und erweitert das Individuum und fördert seine Unabhängigkeit, indem es neue Möglichkeiten zur Ausgestaltung von Selbst und Umwelt sucht und erschafft. Diesem Streben nach Selbstverwirklichung und nach Entfaltung der eigenen Persönlichkeit ist potenziell keine Grenze gesetzt, was in der Bezeichnung Wachstumsbedürfnisse zum Ausdruck gebracht wird.

\subsection{Bedürfnisbefriedigung macht wahrnehmungsfähig}

Der Wunsch nach Respektierung eigener moralischer Vorstellungen und Werte ist ein hochrangiges Wertschätzungsbedürfnis. Der Wunsch nach Gestaltung der Arbeitsumwelt, die auf solchen Werten und Idealen basiert, kann wie auch die ethi- 
sche Reflexion - verstanden als autonome, kritische Reflexion bestehender eigener und neu erfahrener fremder Wertvorstellungen - als Selbstverwirklichungsbedürfnis interpretiert werden.

Betrachtet man die Motivation zur Befriedigung von Wachstumsbedürfnissen unter Berücksichtigung von Defizit- und Progressionsprinzip, führt dies zu einer interessanten Erkenntnis: Erst wenn die vorgeordneten Defizitbedürfnisse angemessen befriedigt sind, besteht für ein Individuum die Möglichkeit, sich überhaupt mit der Befriedigung seiner Wertschätzungs- und Selbstverwirklichungsbedürfnisse zu beschäftigen. Auf die ethische Dimension des klinischen Alltags übertragen, heißt dies: Erst wenn - mindestens - die im Arbeitskontext auftretenden sozialen Bedürfnisse und Sicherheitsbedürfnisse der Mitarbeitenden angemessen befriedigt sind, entsteht eine Bereitschaft, sich auf das Einbringen eigener Wertvorstellungen und auf ethische Reflexion einzulassen.

Umgekehrt formuliert: Kein Mitarbeiter im Krankenhaus fühlt sich intrinsisch motiviert, über ethische Fragestellungen des klinischen Alltags zu reflektieren, wenn im Team keine wertschätzende, anerkennende Grundhaltung herrscht, wenn Kommunikationsprobleme die sozialen Beziehungen dominieren, oder die berufliche Entwicklung im Ungewissen liegt (s. Abb. 15). Die so belasteten Individuen fokussieren ihre Aufmerksamkeit auf das am niedrigsten liegende Defizit und sind bestenfalls motiviert, dieses zu befriedigen, bevor sie überhaupt aus der Wahrnehmung eines darüber liegenden ungestillten Bedürfnisses Motivationskraft gewinnen können. Das schadet sowohl den Mitarbeitenden individuell, der Zusammenarbeit im Team und nicht zuletzt auch den Patienten, für deren Bedürfnisse die Sensibilität verloren geht, wenn die eigenen Grundbedürfnisse nicht befriedigt werden. Die im Titel genannten geordneten Arbeitsverhältnisse beziehen sich auf genau jene Defizitbedürfnis-

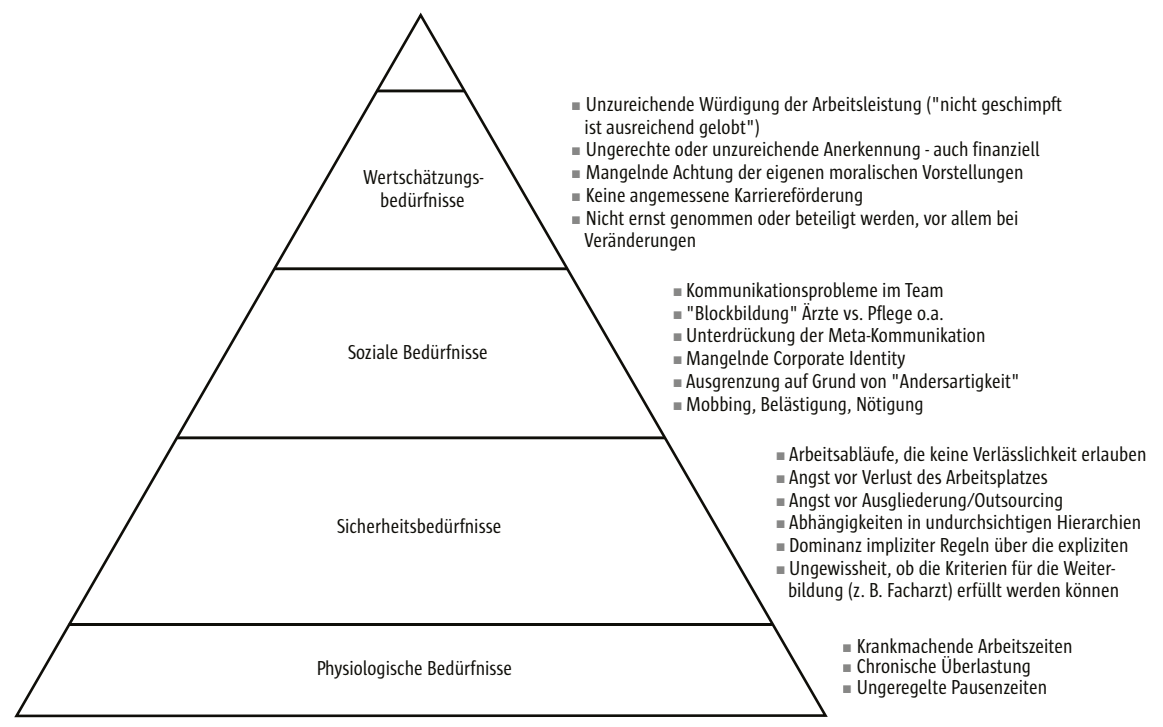

Abb. 15 Einige Probleme des klinischen Arbeitsalltags, die die Beschäftigung mit medizinethischen Anliegen gefährden 
se - Sicherheitsbedürfnisse, soziale Bedürfnisse und weitere Wertschätzungsbedürfnisse -, die befriedigt sein müssen, damit die Mitarbeiter überhaupt einen Sinn in der Beschäftigung mit ethischen Fragestellungen im klinischen Alltag sehen können. Etwas verkürzt mit Brecht gesprochen: Erst kommt das Fressen, dann die Moral.

\subsection{Nur wertgeschätzte Mitarbeitende können mit Patienten wertschätzend umgehen}

Diese Einsicht ist besonders wichtig, wenn klinische Ethikberatung mit dem Ziel implementiert wird oder wurde, bestimmte Aufgaben effizient zu erfüllen, wie sie z. B. die Zentrale Ethikkommission bei der Bundesärztekammer vorschlägt [Neitzke 2008]. Geringe Akzeptanz, Nichtinanspruchnahme oder offene Ablehnung von Ethikberatung oder Gleichgültigkeit ihr gegenüber müssen nicht zwingend auf ein Versagen der Institution während oder nach der Implementierung selbst hinweisen, sondern können ein Anzeichen dafür sein, dass viele Mitarbeiter von ganz anderen Sorgen geplagt werden [Dörries 2008]. Wenn die Mitarbeitenden ihre tägliche Arbeit als Beeinträchtigung ihrer Bedürfnisse oder gar als Wohlfahrtsverlust empfinden, wenn sie von Strukturen innerhalb der Organisation oder Führungspraktiken frustriert werden und zur inneren Emigration übergehen oder gar in großer Zahl den Arbeitsplatz wechseln, hat die Organisation ein viel größeres Problem als die Implementierung klinischer Ethikberatung: Sie verschwendet Mitarbeiter-Ressourcen und ist nicht zur optimalen Patientenversorgung in der Lage. Hier kann eine systemische Organisationsberatung die Mitarbeiter dabei unterstützen, defizitäre Bedürfnisse zu identifizieren und ihre eigenen Lösungen für deren Befriedigung zu finden.

Erst wenn geordnete Arbeitsverhältnisse herrschen, wird eine allgemein akzeptierte klinische Ethikberatung wirksam zur Optimierung von klinischen Prozessen und Strukturen beitragen.

\section{Literatur}

Dörries A (2008) Ethik im Krankenhaus. In: Dörries A, Neitzke G, Simon A, Vollmann I (Hrsg.) Klinische Ethikberatung. Ein Praxisbuch. Kohlhammer, Stuttgart, S. 13-23

Maslow A (1954) Motivation and Personality. New York

Neitzke G (2008) Aufgaben und Modelle von Klinischer Ethikberatung. In: Dörries A, Neitzke G, Simon A, Vollmann I (Hrsg.) Klinische Ethikberatung. Ein Praxisbuch. Kohlhammer, Stuttgart, 58-75 


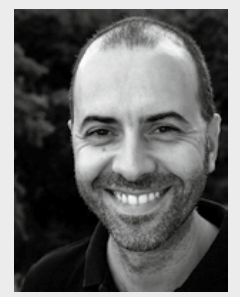

Dipl.-Biol. Dr. biol. hum. Michael Gommel

Herr Dr. Gommel arbeitet seit 1995 als Medizinethiker in Aus-, Fort- und Weiterbildung an Universitäten, Kliniken und Akademien und als Berater für Organisationen im Gesundheitswesen. Seit 2009 hat sich sein Arbeitsschwerpunkt auf die Fortbildung des wissenschaftlichen Nachwuchses und die Beratung von Forschungseinrichtungen in "guter wissenschaftlicher Praxis" verlagert.

Er ist ausgebildeter Systemischer Coach und Prozessberater (die Ausbildung ist vom DBVC anerkannt) und hat eine zertifizierte didaktische Qualifikation. 